\title{
Weekly chemotherapy as first line treatment in frail head and neck cancer patients in the immunotherapy era
}

Andrea Botticelli ${ }^{1+}$, Giulia Pomati ${ }^{2 \dagger}$, Alessio Cirillo ${ }^{3 *}$, Giulia Mammone ${ }^{3}$, Fabio Ciurluini ${ }^{3}$, Bruna Cerbelli ${ }^{3}$, Paolo Sciattella ${ }^{4}$, Massimo Ralli ${ }^{5}$, Umberto Romeo ${ }^{5}$, Francesca De Felice ${ }^{3}$, Carlo Catalano ${ }^{3}$, Francesco Vullo ${ }^{3}$, Marco Della Monaca ${ }^{5}$, Sasan Amirhassankhani ${ }^{6}$, Silverio Tomao ${ }^{3}$, Valentino Valentini ${ }^{5}$, Marco De Vincentiis ${ }^{5}$, Vincenzo Tombolini ${ }^{3}$, Carlo Della Rocca ${ }^{7}$, Antonella Polimeni ${ }^{5}$, Cira di Gioia ${ }^{3}$, Alessandro Corsi ${ }^{3}$, Giulia D'Amati ${ }^{3}$, Silvia Mezi ${ }^{3 \dagger}$ and Paolo Marchetti ${ }^{1 \dagger}$

\begin{abstract}
Objective: First-line therapy for metastatic squamous cell carcinoma of the head and neck (R/M HNSCC) has been revolutionized by the introduction of anti-checkpoint monoclonal antibodies, which have shown a significant improvement in overall survival (OS) gaining approval in a first line setting. Efficacy and safety of first-line weekly chemotherapy, compared to 3-weeks treatment, was retrospectively evaluated in a frail patient population with R/M HNSCC with the aim to evaluate its role as part of a personalized first-line approach.

Methods: A total of 124 patients with locally incurable R/M HNSCC receiving weekly (21) or three-weekly (103) chemotherapy plus cetuximab in a first line setting from December 2010 to September 2020 were retrospectively reviewed. Treatment outcomes in terms of objective response rate (ORR), progression-free survival (PFS), overall survival (OS) and toxicities were analysed.

Results: Patients in the three-week subgroup were ECOG PS 0 (39) and 1 (64) while patients in weekly group (21) were all PS 2. No significant differences were reported in terms of age, sex, smoking and previous alcohol abuse considering the two distinct subgroups. Moreover, no statistically significant difference was found in PFS and OS between the two treatment subgroups. The response rate was 35\% (36 patients) and 34\% (7 patients) in three-week and weekly treatment group, respectively. Seventy patients (68\%) in the three-week group experienced chemotherapyrelated toxicities, predominantly $\mathrm{G} 3$. In the weekly group a predominantly low-grade toxicity was found in a lower number of patients (52\%).
\end{abstract}

Conclusion: The weekly schedule appears to be an active and safe strategy in frail patients with R/M HNSCC. Based on these data, a weekly schedule could be considered as a first line treatment in all frail patients excluded from

\footnotetext{
*Correspondence: alessio.cirillo@uniroma1.it

${ }^{\dagger}$ Andrea Botticelli and Giulia Pomati contributed equally to this work

${ }^{\dagger}$ Silvia Mezi and Paolo Marchetti contributed equally to this work

${ }^{3}$ Department of Radiological, Oncological and Anatomo-Pathological

Science, "Sapienza" University of Rome, Viale Regina Elena 324,

00185 Rome, Italy

Full list of author information is available at the end of the article
}

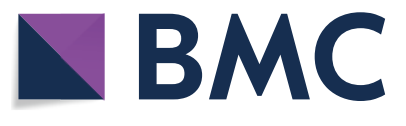

(c) The Author(s) 2021. This article is licensed under a Creative Commons Attribution 4.0 International License, which permits use, sharing, adaptation, distribution and reproduction in any medium or format, as long as you give appropriate credit to the original author(s) and the source, provide a link to the Creative Commons licence, and indicate if changes were made. The images or other third party material in this article are included in the article's Creative Commons licence, unless indicated otherwise in a credit line to the material. If material is not included in the article's Creative Commons licence and your intended use is not permitted by statutory regulation or exceeds the permitted use, you will need to obtain permission directly from the copyright holder. To view a copy of this licence, visit http://creativecommons.org/licenses/by/4.0/. The Creative Commons Public Domain Dedication waiver (http://creativecommons.org/publicdomain/zero/1.0/) applies to the data made available in this article, unless otherwise stated in a credit line to the data. 
pembrolizumab treatment and a study on the combination of weekly chemotherapy and immunotherapy should be performed.

Keywords: Head and neck cancer, Docetaxel, Cetuximab, Chemotherapy, First line, Frail patient population

\section{Introduction}

Head and neck squamous cell carcinoma (HNSCC) represents a heterogeneous spectrum of diseases originating predominantly from oral cavity, oropharynx, hypopharynx, and larynx [1]. HNSCC is globally the sixth most common type of cancer with 830,000 new cases and around 430,000 deaths each year worldwide [2]. Recurrent/metastatic head and neck squamous cell carcinoma (R/M-HNSCC), which is locally incurable, is associated with a poor prognosis [3. The old standard treatment for R/M HNSCC consisted of platinum-based chemotherapy plus cetuximab, according to the therapeutic regimen EXTREME [4]. In a phase III trial, Vermorken et al. demonstrated that adding cetuximab to platinum-5Fluoruracil (5FU) chemotherapy prolonged the median OS and PFS (from 7.4 months to 10.1 months and from 3.3 to 5.6 months, respectively). There were no significant differences in terms of Grade 3 and 4 toxicities between the two arms $(82 \%$ and $76 \%$ in cetuximab and chemotherapy alone arm, respectively); however, in the cetuximab arm there were significantly more cases of sepsis $(p=0.02)$ and hypomagnesemia (0.05) compared to the platinumbased chemotherapy only one [4].

Subsequently, a large randomized trial confirmed the good survival outcomes and response rate of the taxanebased TPE-x regimen observed in the phase II GORTEC study in first line R/M HNSCC [5]. Despite the lack of significant increase of overall survival (OS) when compared to EXTREME, TPEx required a shorter duration (4 vs 6 cycles) with a lower toxicity profile resulting in improvement of quality of life [6]. Furthermore, recent evidences have shown that docetaxel was able to act on the tumour microenvironment favouring the priming of immune response. This mechanism supports the hypothesis that taxanes act synergically with immunotherapy, highlighting the importance of their use in clinical practice [7-10].

A large proportion of patients with R/M HNSCC have poor clinical conditions, weight loss, signs and symptoms related to the extent of the cancer such as pain or obstruction, comorbidities and impaired organ function, therefore they are not suitable for EXTREME treatment due to the high risk of developing high grade toxicity. Conversely, a weekly chemotherapy regimen plus cetuximab was considered a reliable therapeutic strategy in frail cancer patients with R/M-HNSCC [11]. Weekly taxanebased chemotherapy has proved to control cancer growth and its related symptoms, with reduced toxicity and with an appropriate safety profile [12].

First-line therapy for R/M-HNSCC has been revolutionized by the introduction of immune-checkpoint monoclonal antibodies inhibitors (ICIs), a class of drugs targeting the inhibitory immune-checkpoint receptors. The results of the open label randomized phase 3 study KEYNOTE-048 led to approval of the anti PD-1 pembrolizumab in a first line setting, alone or in combination with cisplatin/5 fluorouracil-based chemotherapy [13]. The study evidenced a significantly prolonged OS vs. EXTREME regimen in patients with PD-L1 combined positive score (CPS) $>/=20$ and $C P S>/=1$. Toxicity profile was favourable for pembrolizumab vs. EXTREME and comparable vs. EXTREME for pembrolizumab plus chemotherapy [13].

Nevertheless, some issues are still to be tackled. The KEYNOTE-048 study enrolled patients in good general condition and with ECOG Performance Status (PS) $=0 / 1$, although R/M HNSCC with poor clinical condition $(\mathrm{PS}=2)$ and related signs/symptoms were excluded from this clinical changing study, making its results difficult to be extended to a population of frail patients; in this context the combination strategy still remains an unresolved issue.

This study retrospectively observed the clinical outcomes and toxicities of patients with poor baseline clinical condition treated in a first line setting with a weekly taxane-based chemotherapy, compared to patients with good PS, treated with an EXTREME like chemotherapy regimen.

\section{Materials and methods \\ Patients}

Data from patients with R/M HNSCC who received first line chemotherapy in association with cetuximab in our center from December 2010 to September 2020 were retrospectively analysed.

Patients were clinically staged with contrast enhanced computerized tomography (CT) scan and magnetic resonance imaging (MRI) before starting first line chemotherapy. All patients were discussed and judged as non-eligible for local/regional treatments by the multidisciplinary team of our hospital. Data including age, sex, ECOG PS, comorbidities, history of tobacco smoking and alcohol abuse, primary tumor sites, site of relapse (local/ regional vs. metastatic) and chemotherapy treatment 
schedule were retrospectively collected. On the basis of PS, related symptoms, age, nutritional status and comorbidities, patients were judged as either frail or clinically fit and therefore scheduled for the two different treatments (EXTREME regimen or weekly chemotherapy).

Patients who had comorbidities contraindicating platinum-based chemotherapy and/or cetuximab were not included in the analysis, as well as all patients deemed unsuitable for chemotherapy who have undergone best supportive care.

\section{Treatment and assessments}

Three weekly chemotherapy sessions, according to the EXTREME regimen of cisplatin at a dose of $100 \mathrm{mg} /$ $\mathrm{m}^{2}$ of body-surface area on day 1 , or carboplatin at an area under the curve (AUC) of $5 \mathrm{mg} / \mathrm{ml} /$ minute on day 1 , plus fluorouracil (5-FU) at a dose of $1000 \mathrm{mg} / \mathrm{m}^{2} /$ day for 4 days, plus cetuximab at a dose of $400 \mathrm{mg} / \mathrm{m}^{2}$ as a loading dose, followed by a dose of $250 \mathrm{mg} / \mathrm{m}^{2}$ per week every 21 days for a maximum of 6 cycles, were administered to patients considered fit at the baseline clinical evaluation. Cetuximab maintenance was performed in all patients who achieved at least stable disease (SD) as their best response.

Weekly chemotherapy according to the modified schedule of paclitaxel $80 \mathrm{mg} / \mathrm{m}^{2}$ on day $1,8,15$, carboplatin AUC 2 on day 1, 8, 15 and cetuximab at a $400 \mathrm{mg}$ loading dose followed by $250 \mathrm{mg} / \mathrm{m}^{2}$ weekly every 21 days (PCC), were administered intravenously to those patients deemed frail and unfit for the EXTREME regimen.

Tumor response was assessed every 12 weeks using Response Evaluation Criteria in Solid Tumors (RECIST) guidelines and classified as complete response (CR), partial response (PR), SD, and progressive disease (PD). Toxicities were recorded at day 1 of every cycle and classified according to the National Cancer Institute Common Terminology Criteria for Adverse Events (version 4.0). Progression-free survival (PFS) was defined as the time from the administration of treatment until the first progression or treatment death. The OS was defined as the time from patient registration to death from any cause.

\section{Statistical analysis}

In the descriptive analysis quantitative variables were described as median and range, while qualitative variables were reported as number and percentage. The association between each clinical/pathological feature and outcome was evaluated using univariate and multivariable logistic regression models. Statistical significance was set at $\mathrm{p}<0.05$. All analyses were performed using SAS 9.4 (SAS Institute Inc., Cary, NC, USA).

\section{Results}

Patients

A total of 124 patients with locally incurable $\mathrm{R} / \mathrm{M}$ HNSCC treated with first line platinum-based chemotherapy in association with cetuximab were included in this retrospective study. The baseline clinical/pathological features are reported in Table 1.

$89(72 \%)$ patients were male and median age was 68.4 (SD 11) years. Baseline ECOG PS, evaluated before the start of chemotherapy, was 0,1 , and 2 in 39 (32\%), 64 $(52 \%)$ and $21(17 \%)$ patients, respectively. The primary tumor site was the oropharynx in 18 patients (15\%), the hypopharynx in 8 patients (6\%), the larynx in 40 patients (32\%), the oral cavity in 50 patients $(40 \%)$, and the paranasal sinus in 8 patients (7\%). Histological type was squamous cell carcinoma in all the patients. Human papilloma virus (HPV) status was positive in $2 / 20$ oropharyngeal cancer patients.

Continued smoking during treatment as well as previous alcohol abuse were reported in $26(21 \%)$ and 33 (27\%) of patients, respectively. The site of relapse was locoregional, metastatic or both in 50 (40\%), 59 (48\%) and $15(12 \%)$ of patients, respectively. 103 patients (83\%) received standard first line platinum-based chemotherapy, according to the phase III EXTREME trial, while 21 patients (17\%) received first line PCC weekly chemotherapy.

As shown in Table 1, no significant difference was reported in terms of age, sex, smoking and previous alcohol abuse between the two distinct subgroups of patients based on the received chemotherapy schedule. On the other hand, patients in the EXTREME group were ECOG PS 0 (39) and 1 (64) while patients (21) in the PCC scheme group were all PS 2.

Table 1 Clinical features

\begin{tabular}{lllll}
\hline Parameter & EXTREME & PCC schedule & Total & p value \\
\hline $\mathrm{N}$ & 103 & 21 & 124 & \\
Age, mean (SD) & $68.2(11.1)$ & $69.3(10.8)$ & $68.4(11.0)$ & 0.6798 \\
$<65$ & $33(32.0)$ & $6(28.6)$ & $39(31.5)$ & 0.947 \\
$65-75$ & $41(39.8)$ & $9(42.9)$ & $50(40.3)$ & \\
$>75$ & $29(28.2)$ & $6(28.6)$ & $35(28.2)$ & \\
Male & $72(69.9)$ & $17(81.0)$ & $89(71.8)$ & 0.427 \\
Alcohol & $29(28.2)$ & $4(19.0)$ & $33(26.6)$ & 0.588 \\
Smoke during treat- & $22(21.4)$ & $4(19.0)$ & $26(21.0)$ & 1.000 \\
$\quad$ ment & & & & \\
Baseline PS & & & & \\
0 & $39(37.9)$ & $0(0)$ & $39(31.5)$ & $<0.0001$ \\
1 & $64(52.4)$ & $0(0)$ & $64(52.4)$ & \\
2 & 0 & $21(100)$ & $21(26.04)$ & \\
Toxicity all G & $70(68.0)$ & $11(53.0)$ & $70(56.5)$ & $<0.0001$ \\
\hline
\end{tabular}


70 patients (68\%) in the EXTREME group experienced chemotherapy related toxicity. High grade (G3) neutropenia, anemia and gastrointestinal toxicity was reported in 23 (22\%), 13 (13\%) and 9 patients (9\%) respectively, in accordance with the expected strong toxicity profile.

In the weekly PCC group 11 patients (52\%) reported G1 anemia, 8 patients (40\%) reported G2 neutropenia and 10 patients (49\%) reported G1 gastrointestinal toxicity. No G3-4 toxicities were reported.

\section{Outcomes}

PFS was 4 months (range 1-20) in the overall population; median PFS was 4 and 5 months in the EXTREME and PCC schedule groups, respectively. No statistically significant difference was found in PFS between the two treatment groups $(p=0.275$, Fig. 1$)$. On the univariate analysis (Table 2), no clinical features, including the type of treatment regimen, were associated with the PFS.

OS was 12 months (range 1-50) in the overall population; median OS was 12 months both in the EXTREME and PCC schedule groups. No statistically significant difference was found in OS between the two treatment subgroups ( $p=0.400$, Fig. 2$)$. On the univariate analysis (Table 3), none of the clinical features, including the type of treatment regimen, were found to be predictive of survival.

The response rate was $35 \%$ in EXTREME group (36 patients) and $34 \%$ in PCC group (7 patients) with a relevant palliative effect. None of the patients of both groups achieved a CR.

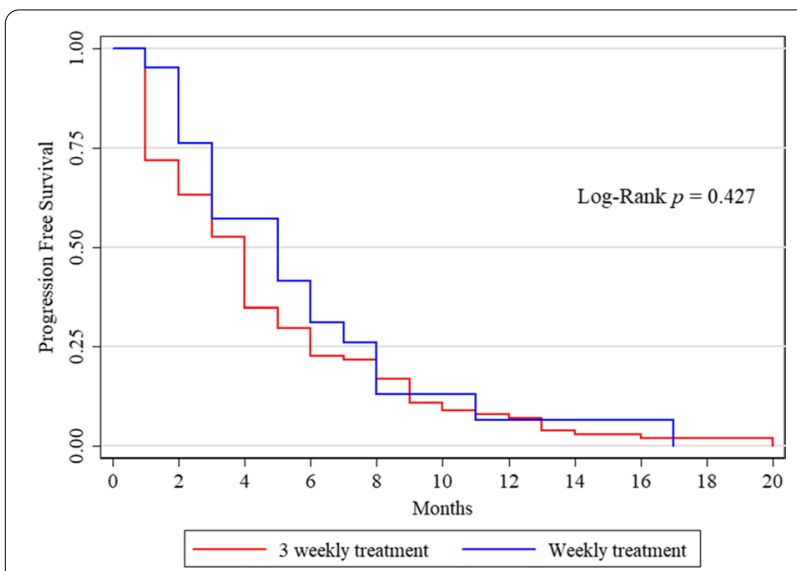

Fig. 1 Kaplan Meyer curves. No statistically significant difference between the EXTREME and PCC schedule subgroups was reported in terms of PFS ( $p$ value 0.427 )
Table 2 Univariate Cox analysis: association between clinical characteristics and PFS

\begin{tabular}{lllll}
\hline Parameter & HR & \multicolumn{2}{l}{$\mathbf{9 5 \%} \mathbf{~ H R ~ C l}$} & p value \\
\hline Gender (m vs f) & 0.98 & 0.66 & 1.47 & 0.935 \\
Age & & & & \\
$\quad<65$ & 1.00 & 1.00 & 1.00 & \\
$65-75$ & 0.87 & 0.57 & 1.34 & 0.526 \\
$>75$ & 1.06 & 0.67 & 1.69 & 0.797 \\
Alcohol & 1.16 & 0.77 & 1.73 & 0.484 \\
Smoke & 0.95 & 0.61 & 1.48 & 0.804 \\
Baseline PS & & & & \\
0 & 1.00 & 1.00 & 1.00 & \\
1 & 1.12 & 0.74 & 1.69 & 0.598 \\
2 & 1.00 & 0.62 & 1.62 & 0.992 \\
Toxicity & 0.77 & 0.53 & 1.12 & 0.169 \\
PTC vs Extreme) & 0.48 & 0.84 & 0.51 & 0.505 \\
\hline
\end{tabular}

\section{Discussion}

In this retrospective study frail patients treated with the PCC chemotherapy regimen had similar PFS compared to fit patients treated with the EXTREME schedule $(p=0.275)$ in a first line setting. These data support the concept of modulating the chemotherapy schedule based on the patient's general condition as a reasonable and safe approach, which does not compromise disease control in frail patients.

In a daily clinical practice setting the coexistence of several factors often makes patients with locally incurable R/M HNSCC fragile and difficult to manage: they often have an ECOG PS > 1, advanced age, severe symptoms and relevant comorbidities (such as diabetes mellitus type 2, arterial hypertension and cardiopathies)

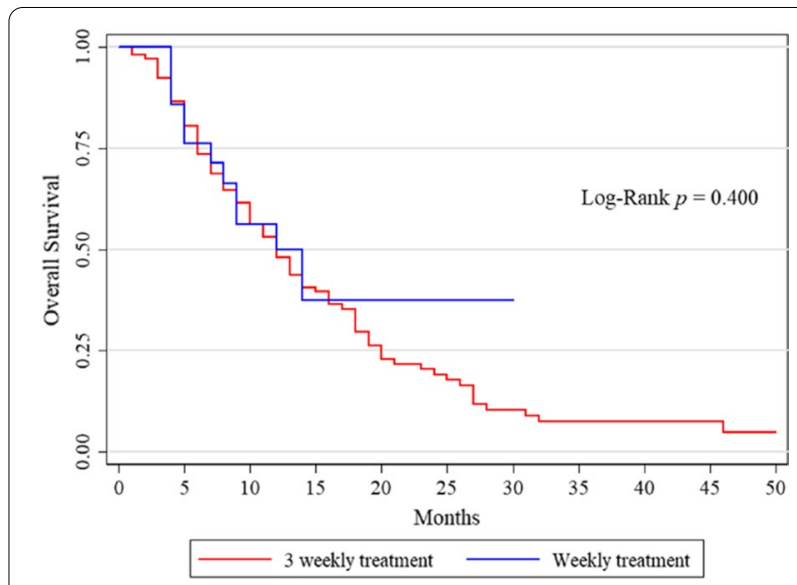

Fig. 2 Kaplan Meyer curves. No statistically significant differences between the EXTREME and PTC schedule subgroups were reported, in terms of OS in our patient population ( $p$ value 0.400 ) 
Table 3 Univariate Cox analysis: association between clinical characteristics and OS

\begin{tabular}{lllll}
\hline Parameter & HR & \multicolumn{2}{l}{$\mathbf{9 5 \%} \mathbf{H R ~ C l}$} & p value \\
\hline Gender (m vs f) & 1.09 & 0.70 & 1.70 & 0.690 \\
Age & & & & \\
$\quad<65$ & 1.00 & 1.00 & 1.00 & \\
$65-75$ & 0.77 & 0.47 & 1.24 & 0.282 \\
$>75$ & 1.03 & 0.63 & 1.70 & 0.894 \\
Alcohol & 1.30 & 0.84 & 2.02 & 0.244 \\
Smoke & 0.93 & 0.57 & 1.52 & 0.778 \\
Baseline PS & & & & \\
0 & 1.00 & 1.00 & 1.00 & \\
1 & 0.91 & 0.58 & 1.42 & 0.675 \\
2 & 0.98 & 0.58 & 1.68 & 0.951 \\
Toxicity & 1.21 & 0.79 & 1.85 & 0.375 \\
PCC vs. Extreme & 0.78 & 0.42 & 1.43 & 0.416 \\
\hline
\end{tabular}

which often limit the use of chemotherapy. Moreover, patients with R/M HNSCC are characterized by frequent malnutrition and poor habits and social status [14]. Consequently, there is an urgent need to customize the therapeutic approach in order to decrease toxicities and morbidities without compromising the oncological outcomes.

In contrast to the PS $0-1$ populations often included in randomized clinical trials, the EXTREME regimen is contraindicated in PS 2 patients, who often make up the majority of the patients seen in real-world clinical practice for the high risk of infectious diseases and high-grade toxicities. Therefore, cetuximab was evaluated in combination with different platinum-based regimens $[15,16]$. Several retrospective and prospective studies have examined first-line chemotherapy based on a weekly combination of paclitaxel and cetuximab in R/M-HNSCCs, showing promising activity $[11,17,18]$. This schedule has proven to be a relevant therapeutic option for patients with poor prognosis considered unsuitable for the EXTREME regimen [17].

In a retrospective study the combination of paclitaxel and cetuximab significantly prolonged the PFS compared to the EXTREME regimen, mostly in older male patients and in patients with tracheostomy [18].

Pêtre et al. evaluated the activity and safety of the weekly carboplatin and paclitaxel chemotherapy in patients with R/M HNSCC unfit for cisplatin due to comorbidities and poor baseline clinical condition. Weekly chemotherapy was efficient and well tolerated in this subgroup of particularly fragile patients [11].

In this study the PCC chemotherapy regimen, used in a population with poor baseline clinical conditions showed a non-inferior efficacy profile, with an excellent tolerability compared to the EXTREME regimen administered in PS 0-1 fit patients. Furthermore, the safety profile was optimal with no high-grade toxicity, compared to the heavy toxicity profile reported in the EXTREME group.

The efficacy is probably due to this optimal tolerability profile, demonstrated by the low degree of toxicity, which allowed an optimal dose intensity even in this group of frail patients. Tolerability could be correlated to the flexibility of the weekly treatment which allows to delay and/ or reduce the dose as needed. Our data are in line with the ones from Naverson et al. who have proved that the use of PCC in patients affected by R/M-HNSCC with PS 0-2 appears to be a profitable therapeutic strategy with low toxicity [19]. This retrospective analysis highlighted that $41 \%$ of patients showed PR or CR and $34 \%$ of patients showed SD. Similarly, in our experience the PCC chemotherapy regimen obtained $34 \%$ of ORR, demonstrating a relevant cytoreductive effect compared to the EXTREME one.

Recently, the phase III Keynote 048 study led to the approval of pembrolizumab, either alone or in combination with platinum/5FU chemotherapy in first line in R/M HNSCCs expressing PD-L1, showing an advantage in terms of OS when compared to standard EXTREME regimen in patients with tumours expressing a combined proportion score (CPS) of 1 or higher ${ }^{13}$. Pembrolizumab in monotherapy could be the first choice in frail patients, since they will not be able to receive chemo/immunotherapy due to the high toxicity rate of the combination. However, the Keynote 048 study included a population of PS 0-1 patients with no comorbidities. Therefore, patients with poor clinical conditions and comorbidities were not represented in this clinical trial and unfortunately there is no data regarding the association of pembrolizumab with weekly platinum-based chemotherapy schedule.

The PCC schedule could be considered as a first line treatment in all frail patients excluded from pembrolizumab treatment, for its optimal efficacy and toxicity profile or in relation to CPS $<1$ or due to the presence of clinical contraindications to immunotherapy treatment such as in autoimmune diseases or uncontrolled infections. A third group of patients which could potentially be a candidate for the PCC treatment is represented by those with frail condition requiring a rapid tumour response, as HNSCC is an aggressive disease often associated to rapid worsening symptoms, such as pain, breathing and feeding difficulties (Fig. 3). In these first and third fragile patient groups immunotherapy with the anti-PD-1 nivolumab can be considered in second-line platinum-resistant disease, allowing for a rational therapeutic sequence [20]. 


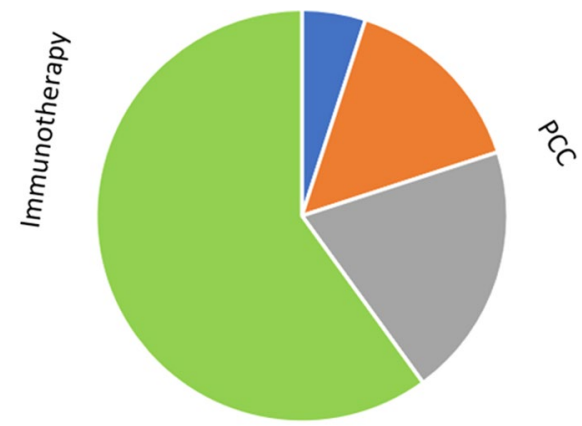

= 5\% Patients who have contraindications to immunotherapy treatment

=15\% Patients excluded for low PD-L1 expression

$=20 \%$ Patiens who require a rapid cytoreductive response

$=60 \%$ Patients who can undergo immunotherapy

Fig. 3 First line in frail patients with R/M HNSCC. Weekly chemotherapy treatment is placed in the group of patients excluded from immunotherapy treatment for low PD-L1 expression (15\%), for contraindications to immunotherapy treatment for uncontrolled infectious or autoimmune diseases or organ transplantation (5\%) or requiring a rapid cytoreductive response (20\%)

This study has some limitations due to its monocentric and retrospective nature. On the other hand, its strength lies on the evaluation of a frail population which is usually excluded from randomized clinical trials. The challenge is to define a customized first-line approach in head and neck cancer patients without excluding frail patients, considering patient preferences, socio-economic status and the availability of caregivers. Each treatment should be determined considering PS and comorbidity, symptoms and CPS, the need of rapid response, risk of complications and site of relapse [21], necessarily in the context of a multidisciplinary team evaluation. This tailored approach could lead to excellent oncological outcomes, gaining disease control without compromising the patient's quality of life.

In conclusion, a PCC schedule appears indicated in frail patients and could currently be considered in all frail patients excluded from immunotherapy treatment $(\mathrm{CPS}<1)$ or when immunotherapy treatment has contraindicated as well as when a prompt and strong response is required for disease control in critical sites. It also appears advisable to explore the combination of check point inhibitors with modulated chemotherapy regimens in frail patients in the near future.

Weekly chemotherapy treatment is placed in the group of patients excluded from immunotherapy treatment for low PD-L1 expression (15\%), for contraindications to immunotherapy treatment for uncontrolled infectious or autoimmune diseases or organ transplantation $(5 \%)$ or requiring a rapid cytoreductive response (20\%).
Acknowledgements

Not applicable.

\section{Authors' contributions}

Conception and design: $A B, S M$ and PM. Manuscript writing: $A B$ and GP. Data acquisition: GP and AC (Alessio Cirillo). Analysed the data: PS. Discussion of the results and implications of findings: $A B, P M, S M$ and $G P$. Data interpretation: $A B, S M, G P$ and $A C$ (Alessio Cirillo). Drafting of the manuscript: $A B, G P$ and $S M$. Revising the content: GM, FC, BC, MR, UR, FDF, CC, FV, MDM, SA, ST, W, MDV, VT, CDR, AP, CDG, AC, GDA, AC (Alessio Cirillo) and SM. All authors read and approved the final manuscript.

\section{Funding}

This research received no external funding.

\section{Availability of data and materials}

All data generated or analysed during this study are included in this published article [and its supplementary information files].

\section{Declarations}

Ethics approval and consent to participate

All patients provided a written informed consent, and protocol approval of the Local Ethics Committee was obtained [CE 5618].

\section{Consent for publication}

Not applicable.

\section{Competing interests}

Paolo Marchetti (PM) has a consultant/advisory role for BMS, Roche-Genentech, MSD, Novartis, Amgen, Merck Serono, Pierre Fabre, and Incyte. The other Authors declare that the research was conducted in the absence of any commercial or financial relationship which could be construed as a potential conflict of interest.

\section{Author details}

1 'Department of Clinical and Molecular Oncology, "Sapienza" University of Rome, 00185 Rome, Italy. Department of Molecular Medicine, "Sapienza" University of Rome, 00185 Rome, Italy. 'Department of Radiological, Oncological and Anatomo-Pathological Science, "Sapienza" University of Rome, Viale Regina Elena 324, 00185 Rome, Italy. ${ }^{4}$ Department of Statistical Sciences, Sapienza University of Rome, 00161 Rome, Italy. ${ }^{5}$ Odontostomatological and Maxillo-Facial Science, 'Sapienza' University of Rome, 00185 Rome, Italy. ${ }^{6}$ MSc Guy's \& St Thomas' NHS Foundation Trust, Westminster Bridge Rd, Bishop's, London SE1 7EH, UK. 7 Department of Medico-Surgical Sciences and Biotechnology, Sapienza University, Polo Pontino, 00185 Rome, Italy.

Received: 18 May 2021 Accepted: 2 July 2021

Published online: 12 July 2021

References

1. Johnson DE, Burtness B, Leemans CR, Lui VWY, Bauman JE, Grandis JR. Head and neck squamous cell carcinoma. Nat Rev Dis Primers. 2020;6(1):92. https://doi.org/10.1038/s41572-020-00224-3.

2. Yitian L, Deshi D. Cetuximab plus chemotherapy versus chemotherapy alone in recurrent or metastatic head and neck squamous cell carcinoma: a cost-effectiveness analysis. Cancer Manag Res. 2020;6(12):11383-90. https://doi.org/10.2147/CMAR.S272149.

3. Yoo SH, Ock CY, Keam B, Park SJ, Kim TM, Kim JH, Jeon YK, Chung EJ, Kwon SK, Hah JH, Kwon TK, Jung KC, Kim DW, Wu HG, Sung MW, Heo DS. Poor prognostic factors in human papillomavirus-positive head and neck cancer: who might not be candidates for de-escalation treatment? Korean J Intern Med. 2019;34(6):1313-23. https://doi.org/10.3904/kjim.2017.397.

4. Vermorken JB, Mesia R, Rivera F, Remenar E, Kawecki A, Rottey S, Erfan J, Zabolotnyy D, Kienzer HR, Cupissol D, Peyrade F, Benasso M, Vynnychenko I, De Raucourt D, Bokemeyer C, Schueler A, Amellal N, Hitt R. 
Platinum-based chemotherapy plus cetuximab in head and neck cancer. N Engl J Med. 2008;359(11):1116-27. https://doi.org/10.1056/NEJMo a0802656.

5. Guigay J, Fayette J, Dillies AF, Sire C, Kerger JN, Tennevet I, Machiels JP, Zanetta S, Pointreau Y, Bozec Le Moal L, Henry S, Schilf A, Bourhis J. Cetuximab, docetaxel, and cisplatin as first-line treatment in patients with recurrent or metastatic head and neck squamous cell carcinoma: a multicenter, phase II GORTEC study. Ann Oncol. 2015;26:1941-7. https:// doi.org/10.1093/annonc/mdv268.

6. Guigay J, Fayette J, Mesia R, Lafond C, Saada-Bouzid E, Geoffrois L, Martin L, Cupissol D, Capitain O, Castanie H, Vansteene D, Schafhausen P, Dubos Arvis C, Even C, Sire C, Delhommeau M, Michel C, Bourhis J, Keilholz U, Auperin A. TPExtreme randomized trial: TPEx versus extreme regimen in 1st line recurrent/metastatic head and neck squamous cell carcinoma (R/M HNSCC). J Clin Oncol. 2019;37:6002. https://doi.org/10.1200/JCO. 2019.37.15_suppl.6002.

7. Schmid P, Adams S, Rugo HS, Schneeweiss A, Barrios CH, Iwata H, Diéras V, Hegg R, Im SA, Shaw Wright G, Henschel V, Molinero L, Chui SY, Funke R, Husain A, Winer EP, Loi S, Emens LA. Atezolizumab and Nab-Paclitaxel in advanced triple-negative breast cancer. N Engl J Med. 2018;379:2108-21. https://doi.org/10.1056/NEJMoa1809615.

8. Kang C, Syed YY. Atezolizumab (in combination with Nab-paclitaxel): a review in advanced triple-negative breast cancer. Drugs. 2020;80(6):6017. https://doi.org/10.1007/s40265-020-01295-y.

9. Hu Y, Liu J, Cui P, Liu T, Piao C, Xu X, Zhang O, Xiao M, Lu Y, Liu X, Wang Y, Lu X. Synergistic effect of adoptive immunotherapy and docetaxel inhibits tumor growth in a mouse model. Cell Immunol. 2020;348: 104036. https://doi.org/10.1016/j.cellimm.2019.104036.

10. Bernichon E, Tissot C, Bayle-Bleuez S, Rivoirard R, Bouleftour W, Forest F, Tinquaut F, Mery B, Fournel P. Predictive resistance factors in lung cancer patients treated with Nivolumab. Retrospective study. Bull Cancer. 2021;108(3):250-65. https://doi.org/10.1016/j.bulcan.2020.10.010.

11. Pêtre A, Dalban C, Karabajakian A, Neidhardt EM, Roux PE, Poupart M, Deneuve S, Zrounba P, Fayette J. Carboplatin in combination with weekly Paclitaxel as first-line therapy in patients with recurrent/metastatic head and neck squamous cell carcinoma unfit to EXTREME schedule. Oncotarget. 2018;9(31):22038-46. https://doi.org/10.18632/oncotarget.

12. Tousif D, Sarathy V, Kumar R, Naik R. Randomized controlled study comparing efficacy and toxicity of weekly vs. 3-weekly induction chemotherapy in locally advanced head and neck squamous cell carcinoma. Front Oncol Front Oncol. 2020;10:1284. https://doi.org/10.3389/fonc.2020. 01284.

13. Burtness $B$, Harrington $K J$, Greil $R$, Soulières $D$, Tahara $M$, de Castro Jr $G$, Psyrri A, Basté N, Neupane P, Bratland Å, Fuereder T, Hughes BGM, Mesía R, Ngamphaiboon N, Rordorf T, Wan Ishak WZ, Hong RL, González Mendoza R, Roy A, Zhang Y, Gumuscu B, Cheng JD, Jin F, Rischin D. Pembrolizumab alone or with chemotherapy versus cetuximab with chemotherapy for recurrent or metastatic squamous cell carcinoma of the head and neck (KEYNOTE-048): a randomised, open-label, phase 3 study. Lancet. 2019;394(10212):1915-28. https://doi.org/10.1016/S0140-6736(19) 32591-7.
14. Gorenc M, Kozjek NR, Strojan P. Malnutrition and cachexia in patients with head and neck cancer treated with (chemo)radiotherapy. Rep Pract Oncol Radiother Jul-Aug. 2015;20(4):249-58. https://doi.org/10.1016/j. rpor.2015.03.001.

15. Trieu V, Pinto H, Riess JW, Lira R, Luciano R, Coty J, Boothroyd D, Dimitrios CA. Weekly docetaxel, cisplatin, and cetuximab in palliative treatment of patients with squamous cell carcinoma of the head and neck. Oncologist. 2018;23(7):764-e86. https://doi.org/10.1634/theoncologist.2017-0618.

16. Hitt R, Irigoyen A, Cortes-Funes H, Grau JJ, García-Sáenz JA, Cruz-Hernandez JJ. Spanish Head and Neck Cancer Cooperative Group (TTCC). Phase Il study of the combination of cetuximab and weekly paclitaxel in the first-line treatment of patients with recurrent and/or metastatic squamous cell carcinoma of head and neck. Ann Oncol. 2012;23(4):1016-22. https://doi.org/10.1093/annonc/mdr367.

17. Tahara M, Kiyota N, Yokota T, Hasegawa Y, Muro K, Takahashi S, Onoe T, Homma A, Taguchi J, Suzuki M, Minato K, Yane K, Ueda S, Hara H, Saijo K, Yamanaka T. Phase II trial of combination treatment with paclitaxel, carboplatin and cetuximab (PCE) as first-line treatment in patients with recurrent and/or metastatic squamous cell carcinoma of the head and neck (CSPOR-HNO2). Ann Oncol. 2018;29(4):1004-9. https://doi.org/10. 1093/annonc/mdy040.

18. Nakano K, Marshall S, Taira S, Sato Y, Tomomatsu J, Sasaki T, Shimbashi W, Fukushima H, Yonekawa H, Mitani H, Kawabata K, Takahashi S. A comparison of weekly paclitaxel and cetuximab with the EXTREME regimen in the treatment of recurrent/metastatic squamous cell head and neck carcinoma. Oral Oncol. 2017;73:21-6. https://doi.org/10.1016/j.oraloncolo gy.2017.07.022.

19. Narveson L, Kathol E, Rockey M, Henry D, Grauer D, Neupane P. Evaluation of weekly paclitaxel, carboplatin, and cetuximab in head and neck cancer patients with incurable disease. Med Oncol. 2016;33(10):107. https://doi. org/10.1007/s12032-016-0822-0.

20. Ferris RL, Blumenschein G Jr, Fayette J, Guigay J, Colevas AD, Licitra L, Harrington K, Kasper S, Vokes EE, Even C, Worden F, Saba NF, Iglesias Docampo LC, Haddad R, Rordorf T, Kiyota N, Tahara M, Monga M, Lynch M, Geese WJ, Kopit J, Shaw JW, Gillison ML. Nivolumab for recurrent squamous-cell carcinoma of the head and neck. N Engl J Med. 2016;375(19):1856-67. https://doi.org/10.1056/NEJMoa1602252.

21. Botticelli A, Cirillo A, Scagnoli S, Cerbelli B, Strigari L, Cortellini A, Pizzuti L, Vici P, De Galitiis F, Di Pietro FR, Cerbelli E, Ghidini M, D'Amati G, Della Rocca C, Mezi S, Gelibter JA, Giusti R, Cortesi E, Ascierto PA, Nuti M, Marchetti P. The agnostic role of site of metastasis in predicting outcomes in cancer patients treated with immunotherapy. Vaccines. 2020;8(2):203. https://doi.org/10.3390/vaccines8020203.

\section{Publisher's Note}

Springer Nature remains neutral with regard to jurisdictional claims in published maps and institutional affiliations.

\footnotetext{
Ready to submit your research? Choose BMC and benefit from:

- fast, convenient online submission

- thorough peer review by experienced researchers in your field

- rapid publication on acceptance

- support for research data, including large and complex data types

- gold Open Access which fosters wider collaboration and increased citations

- maximum visibility for your research: over $100 \mathrm{M}$ website views per year
}

At BMC, research is always in progress.

Learn more biomedcentral.com/submissions 